\title{
Ueber einige optisch-aktive Kobaltsalze mit $\beta$-Diketonresten im Komplex
}

von

\section{A. Werner $\dagger$, Jeanne E. Sehwyzer und Walter Karrer ${ }^{1}$ ).}

(20. XII. 20.)

Die schon früher ausgesprochene Auffassung, dass Metallsalze der $\beta$-Diketone als innere Komplexsalze zu formulieren sind, in welchen ein $\beta$-Diketonrest zwei Koordinationsstellen am Zentralatom besetzt ${ }^{2}$ ), gewann an Wahrscheinlichkeit, als vor drei Jahren an dieser Stelle gezeigt wurde ${ }^{3}$ ), dass der einfachste Vertreter der $\beta$-Diketonklasse, Acetylaceton, sich leicht an Stelle von zwei koordinativ einwertigen Gruppen in einen bekannten Kobaltikomplex einführen lässt. Der Diketonrest übernimmt dabei eine ähnliche Rolle wie die Kohlensäure, Oxalsäure oder Malonsäure in vielen bekannten Komplexsalzen.

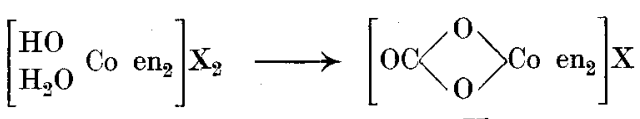

I.

Hydroxo-aquo-diäthylendiamin-

Kobaltisalze

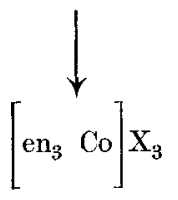

III.

Triäthylendiamin-Kobaltisalze
II.

Karbonato-diäthylendiamin-

Kobaltisalze

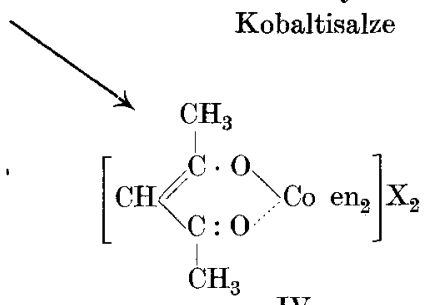

IV.

Acetylacetonato-diäthylendiamin-

Kobaltisalze

Ist diese Auffassung richtig, so muss die letztformulierte Reihe IV infolge von Molekularasymmetrie in zwei optisch-aktiven

1) Vgl. die beiden Diss. Zürich 1919, und Helv. 3, 472. Fussnote 1 (1920).

2) A. Werner, B. 34, 2586 (1901).

3) A. Werner und S. Matissen, Helv. 1, 78 (1918). 
Formen auftreten; dasselbe trifft bekanntlich für die Reihen II und III $\mathrm{zu}^{1}$ ).

Leider gelang die direkte Spaltung der Acetylacetonatodiäthylendiamin-kobaltisalze nicht ${ }^{2}$ ).

Ein ähnliches Verhalten wurde seinerzeit an den Salzen der Karbonato-diäthylendiamin-kobaltireihe II beobachtet; man konnte indessen ihre optisch-aktiven Formen auf synthetischem Wege gewinnen, indem man die Karbonatogruppe in die bereits optisch-aktiven Komponenten eines geeigneten Komplexes einführte; diesen fand man in den d- resp. I-Dichlorodiäthylendiaminkobaltisalzen ${ }^{3}$ ).

Wir sind nun in der Lage, über die erfolgreiche Anwendung einer analogen Methode zur Darstellung der optisch-aktiven Acetylacetonato- resp. Propionylacetonato-diäthylendiaminkobaltisalze zu berichten; der Vollständigkeit hallier wurde auch die bis jetzt unbekannte racemische Propionylacetonatoreihe dargestellt.

Die Hydroxo-aquo-diäthylendiamin-kobaltireihe diente uns in allen Fällen als Ausgangsmaterial. Es erwies sich aber als vorteilhaft, von ihrer Isolierung abzusehen, vielmehr direkt ihre Lösungen zu verwenden, welche aus den 1,2-Chloro-aquo-diäthylendiamin-kobaltisalzen mit der berechneten Menge wässeriger Kalilauge entstehen:

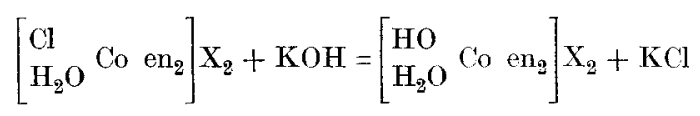

Durch vorsichtiges Erwärmen mit etwas mehr als der berechneten Menge $\beta$-Diketon, erhielt man die neuen Reihen:

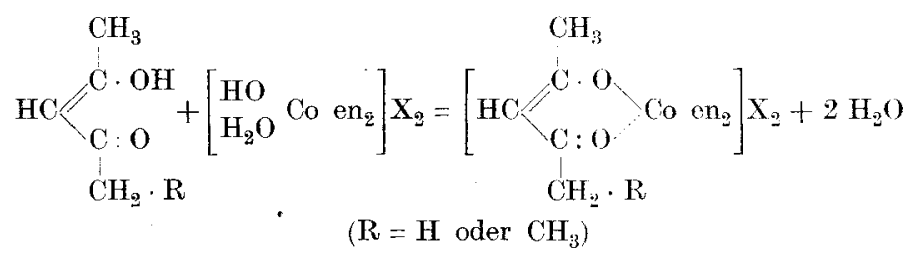

Bei Anwendung der optisch-aktiven Chloro-aquo-diäthylendiamin-kobaltisalze sind die daraus gewonnen Lösungen der (1912).

1) A. Werner, B. 45, 121 (1912); derselbe und Mc. Cutcheon, B. 45, 3282

2) Vgl. Diss. S. Matissen, Zürich 1912, S. 54.

3) A. Werner und Mc. Cutcheon l. e. 
Hydroxo-aquo-salze und die der Acetylacetonato- resp. Propionylacetonato-reihen ebenfalls optisch-aktiv. Ein Teil des Materials wird allerdings racemisiert, und zwar in besonders erheblichem Masse bei der Propionylacetonato-Reihe; doch gelingt es ohne besondere Mühe, die als Jodide isolierten Reihen durch Umkrystallisieren in reine optisch-aktive Fraktionen einerseits, und racemische Fraktionen andererseits zu zerlegen; als Lösungsmittel verwendet man dabei am besten Äthylalkohol, in welchem die erwähnten Jodide noch sehr gut, aber etwas weniger löslich sind, als in Wasser.

Die als Ausgangsmaterial benötigten optisch-aktiven Chloroaquo-diäthylendiamin-kobaltireihen sind durch Spaltung der racemischen Reihe mittelst $\mathrm{d}$-( $\alpha$-Brom-) campher- $\boldsymbol{\pi}$-sulfonsäure zugänglich, worüber später ausführlich berichtet werden soll; die grosse Beweglichkeit des Cl-Atoms und der $\mathrm{H}_{2} \mathrm{O}$-Molekel, die im Komplex dieser Reihen enthalten sind, machen sie zu einem vorzüglichen Material für Synthesen optisch-aktiver Kobaltikomplexe.

Die neu dargestellten Verbindungen sind in ihren äusseren Eigenschaften den schon früher beschriebenen Acetylacetonatodiäthylendiamin-kobaltisalzen recht ähnlich; sie sind gut krystallisierende Körper von granatroter Farbe; zerrieben, stellen sie mennigrote Pulver dar; ihre Lösungen sind leuchtend blutrot. Die $\beta$-Diketonreste sind sehr fest im Komplex gebunden: die Salze lassen sich ohne Schwierigkeit umkrystallisieren, ihre Lösungen können im allgemeinen sogar bis zur Trockne eingedampft werden, ohne dass Zersetzung eintritt.

Sowohl die Salze der Acetylacetonato-reihen als auch diejenigen der Propionylacetonato-reihen sind meistens in Wasser leicht bis sehr leicht löslich; noch leichter lösen sie sich in Aceton, etwas weniger leicht in Äthylalkohol, nicht in Äther. Die Löslichkeiten sind für die letzteren Reihen allgemein grösser als für die ersten; die racemischen Formen sind weniger löslich, als die entsprechenden optisch-aktiven.

Diese letzteren weisen sehr hohe Drehungen auf, welche etwa zweimal so gross sind, wie die der entsprechenden aktiven Chloroaquo-reihen, von welchen die Synthese ihren Ausgang nahm; hingegen bleibt die Drehungsrichtung unverändert. Es besteht eine ausgesprochene anormale Rotationsdispersion mit einem Maximum bei $\mu \mu 554$ (s. Tabelle II im exp. Teil). Die Homologie 
der komplex gebundenen $\beta$-Diketonreste scheint einen nicht unwesentlichen Einfluss auf die Grösse des Drehungsvermögens zu haben: die molekularen Drehungen sind in der Propionylacetonato-reihe (frisch dargestellt) durchweg um etwa $10 \%$ höher als bei den entsprechenden Salzen der Acetylacetonato-reihe (s. Tabelle I), wobei allerdings die Frage offen gelassen werden muss, ob die letzteren optisch ganz rein sind.

Tabelle I.

\begin{tabular}{|c|c|c|}
\hline \multicolumn{2}{|c|}{ d-resp.1- $\left[\mathrm{C}_{5} \mathrm{H}_{7} \mathrm{O}_{2} \mathrm{Co}_{\text {en }}\right]\left(\mathrm{NO}_{3}\right)_{2}$} & d- resp.l- $\left[\mathrm{C}_{6} \mathrm{H}_{9} \mathrm{O}_{2} \mathrm{Coen}_{2}\right]\left(\mathrm{NO}_{3}\right)_{2}$ \\
\hline$[\alpha]_{\mathrm{D}}$ & $\pm 550^{\circ}$ & $+596^{\circ}$ resp. $-591^{\circ}$ \\
\hline$[\mathrm{M}]_{\mathrm{D}}$ & $+2217^{0}$ & $+2481^{\circ}$ resp. $-2460^{\circ}$ \\
\hline
\end{tabular}

Die unreinen, z. B. kleine Mengen von Fällungsmitteln enthaltenden Salze der optisch-aktiven Reihen fallen beim Umkrystallisieren aus Wasser leicht der Racemisierung anheim, während diese in alkoholischer Lösung gehemmt ist. Lösungen der reinen Salze können dagegen bis zur Trockne eingedampft werden, ohne dass sie sich racemisieren. Merkwürdigerweise nimmt das Drehungsvermögen der reinen Propionylacetonatosalze bei Lagerung der Präparate ab, in 4 Monaten um ca. $15 \%$.

\section{Experimenteller Teil. $\left.{ }^{1}\right)$}

I. Optisch-aktive Acetylacetonato-diäthylendiaminkobaltisalze. (J. E. Schwyzer.)

\section{Ausgangsmaterial.}

Das racemische Chloro-aquo-diäthylendiamin-kobaltibromid wurde gemäss den Angaben von $A$. Werner ${ }^{2}$ ) nach folgendem Schema dargestellt:

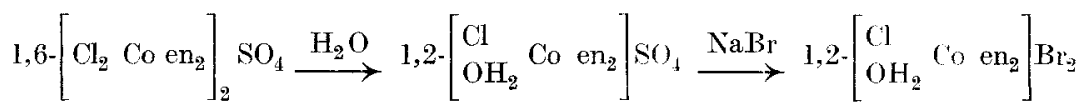

1) Die Vorversuche sind von den Assistenten, den HH. Dr. E. Lïischer und Al. P. Smirnoff ausgeführt worden.

2) A. 386, $122(1912)$. 
Zur Spaltung dieses Salzès in optisch-aktive Komponenten wurde z. B. wie folgt verfahren ${ }^{1}$ ): 150 gr racemisches 1,2 -Chloroaquo-diäthylendiamin - kobaltibromid (Rohprodukt) und $135 \mathrm{gr}$ d-( $\alpha$-brom) campher- $\pi$-sulfonsaures Ammonium werden in $500 \mathrm{~cm}^{3}$ Wasser bei $40^{\circ}$ gelöst. Man filtriert vom geringen Rückstand $a b$ und rührt nun das Filtrat unter Eiskühlung ständig mit einem Glasstab um, wobei nach ca. 3/4 Std. Abscheidung des Bromcamphersulfonates der l-Reihe beginnt. Nachdem die ganze Masse fast fest geworden ist, gibt man noch $100-250 \mathrm{~cm}^{3}$ Eiswasser hinzu, bis eben ein gut filtrierbarer Brei entsteht. Man saugt nun den Niederschlag scharf ab und wäscht ihn mit 70-80 $\mathrm{cm}^{3}$ Eiswasser; hierauf verreibt man den so erhaltenen, rosafarbenen Kuchen mit $170 \mathrm{~cm}^{3}$ Bromwasserstoffsäure $(\mathrm{D}=1,48)$, wobei ein Brei von glänzenden, dunkel-violettroten Kryställchen des l-Bromides sich bildet. Dieses wird abgesaugt, in Wasser bei höchstens $40^{\circ}$ gelöst $\left(5 \mathrm{~cm}^{3}\right.$ Wasser auf $1 \mathrm{gr}$ Salz) und unter Eiskühlung mit einem Drittel seines Volumens an Bromwasserstoffsäure ausgefällt. Das so erhaltene, abfiltrierte und mit Alkohol und Äther gewaschene 1-Bromid ist meistens rein. Seine wässerige Lösung $\left(1 \%\right.$ ) zeigt die spezifische Drehung von $[\alpha]_{\mathrm{D}}=-214^{\circ}$. Ist dieser Reinheitsgrad nicht erreicht, so wiederholt man die soeben beschriebene Reinigung, bis das reine l-Bromid vorliegt.

Das Filtrat vom Bromcamphersulfonat der l-Reihe wird unter Eiskühlung mit einem Drittel seines Volumens $\left(200-250 \mathrm{~cm}^{3}\right)$ an Bromwasserstoffsäure $(d=1,48)$ versetzt. Nach einiger Zeit scheidet sich das d-Bromid aus, welches abfiltriert und in ähnlicher Weise wie das 1-Salz gereinigt wird. Die spezifische Drehung des reinen d-Bromides ist ebenso gross, wie diejenige des 1-Salzes: $[\alpha]_{\mathrm{D}}=+214^{\circ}$. Man erhält bei obigem Ansatz 35-40 gr von jeder Form; die beiden Bromide stellen schöne, dunkel violettrote Prismen dar, welche eine Molekel Krystallwasser enthalten.

\section{Darstellung der optisch-altiven Acetylacetonato-diäthylendiamin- kobaltisalze.}

$3 \mathrm{gr} \mathrm{d}$ - bezw. 1-Chloro-aquo-bromid werden mit $6 \mathrm{~cm}^{3} 10$-proz. Kalilauge übergossen, wobei sich das Salz in der Regel mit rotvioletter Farbe auflöst. Man fügt $1,5 \mathrm{~cm}^{3}$ Acetylaceton, gelöst in $4 \mathrm{~cm}^{3}$ Alkohol, hinzu und erwärmt etwa eine Minute lang auf

1) Vgl. auch H. Fischlin, Diss. Zürich, 1919. 
$40^{\circ}$, wobei die Lösung eine intensive, orangestichig-rote Farbe annimmt. Man filtriert und versetzt das Filtrat mit ca. $5 \mathrm{gr}$ pulverisiertem Kaliumjodid. Das dadurch ausgeschiedene leuchtend rote Jodid wird abgesaugt und auf Ton getrocknet; eine weitere Menge erhält man beim Süttigen des Filtrats mit Kaliumjodid.

Man erhält so $4-5$ gr Jodid der aktiven Acetylacetonatodiäthylendiamin-kobaltireihe der gleichen Drehungsrichtung, wie sie das angewendete Chloro-aquobromid reigt; jedoch ist das Salz nicht optisch rein, sondern mit wechselnden Mengen racemisiertem Salz vermischt.

Zur Reinigung löst man das Rohjodid in wenig siedendem Alkohol und lässt die rasch filtrierte Lösung langsam erkalten; dabei scheidet sich das reine d- resp. 1-Jodid in feinen nadeligen Krystallen ab, die abfiltriert und auf Ton getrocknet werden.

Die 0,1-proz. wässerigen Lösungen der reinen Jodide zeigen die spezifische Drehung $[a]_{\mathrm{D}}= \pm 400^{\circ}$.

Aus der Mutterlauge dieser Fraktionen werden beim längeren Stehen weitere, jedoch viel schwächer drehende Jodidmengen erhalten.

Salze der optisch-aktiven Acetylacetonato-diäthylendiaminkobaltireihen.

J o di d e: d- resp. 1- $\left[\mathrm{C}_{5} \mathrm{H}_{7} \mathrm{O}_{2}\right.$ Co en 2$\left.]\right]_{2}+1 \mathrm{H}_{2} \mathrm{O}$

Die in der bereits geschilderten Weise dargestellten und aus Alkohol umkrystallisierten optisch-aktiven Jodide bilden dünne, granatrote Prismen, welche eine Molekel Krystallwasser enthalten, sich sehr leicht in Wasser, etwas weniger in Alkohol und spielend in Aceton lösen.

a) d-Jodid: $32,888 \mathrm{mgr}$ Subst. gaben 1,095 mgr $\mathrm{H}_{2} \mathrm{O}\left(105^{0}\right)$

38,199 mgr Subst. gaben $10,83 \mathrm{mgr} \mathrm{CoSO}_{4}$

14,956 mgr Subst. gaben 12,72 mgr AgJ

$13,885 \mathrm{mgr}$ Subst. gaben $10,65 \mathrm{mgr} \mathrm{CO}_{2}$ und $6,00 \mathrm{mgr}_{2} \mathrm{O}$

$7,990 \mathrm{mgr}$ Subst. gaben $0,7203 \mathrm{~cm}^{3}$ (korr.) $\mathrm{N}_{2}\left(13^{0}, 719 \mathrm{~mm}\right)$.

$\mathrm{C}_{9} \mathrm{H}_{23} \mathrm{O}_{2} \mathrm{~N}_{4} \mathrm{~J}_{2} \mathrm{Co}+\mathrm{H}_{2} \mathrm{O}$

Ber. C 19,60 H 4,71 N 10,17 J 46,06 Co $10,70 \quad \mathrm{H}_{2} \mathrm{O} \quad 3,26_{\%}^{\circ}$

Gef. , $19,97,4,85 \quad, 10,18 \quad, 45,94 \quad, 10,78 \quad, \quad 3,32 \%$ 
b) 1-Jodid: 48,116 mgr Subst. gaben 1,616 mgr $\mathrm{H}_{2} \mathrm{O}\left(105^{0}\right)$

47,790 mgr Subst. gaben 13,38 mgr $\mathrm{CoSO}_{4}$

14,712 mgr Subst. gaben $12,66 \mathrm{mgr} \mathrm{AgJ}$

10,320 mgr Subst. gaben 7,67 mgr $\mathrm{CO}_{2}$ und 4,54 mgr $\mathrm{H}_{2} \mathrm{O}$

7,821 mgr Subst. gaben $0,6958 \mathrm{~cm}^{3}$ (korr.) $\mathrm{N}_{2}\left(13^{0}, 723 \mathrm{~mm}\right.$ )

$\mathrm{C}_{9} \mathrm{H}_{23} \mathrm{O}_{2} \mathrm{~N}_{4} \mathrm{~J}_{2} \mathrm{Co}+\mathrm{H}_{2} \mathrm{O}$

Ber. C 19,60 H 4,71 N 10,17 J 46,06 Co $10,70 \quad \mathrm{H}_{2} \mathrm{O} 3,26 \%$

Gef. , $20,27,4,93,10,11,46,52,10,65 \quad, 3,35 \%$.

Polarisationen. Es wurden hier und auch in den folgenden Beispielen 0,1proz. wässerige Lösungen der betreffenden Salze verwendet; die Schichtlänge betrug $10 \mathrm{~cm}$.

$$
\alpha_{\mathrm{D}}= \pm 0,4 ;[\alpha]_{\mathrm{D}}= \pm 400^{\circ} ;[\mathrm{M}]_{\mathrm{B}}= \pm 2204^{\circ} .
$$

Aus den Jodiden können die anderen Salze der beiden Reihen dargestellt werden, und zwar entweder durch direkten doppelten Umsatz, oder auf dem Umwege über die ausserordentlich leicht löslichen Chloride.

\section{Chloride: d-resp.l- $\left[\mathrm{C}_{5} \mathrm{H}_{7} \mathrm{O}_{2} \mathrm{Coen}_{2}\right] \mathrm{Cl}_{2}+1 \mathrm{H}_{2} \mathrm{O}$}

Reines d- resp. l-Jodid wird in absolut alkoholischer Lösung mit überschüssigem trockenem Silberchlorid so lange geschüttelt, bis eine abfiltrierte Probe durch Silbernitrat rein weiss gefällt wird. Man filtriert die Lösung, kühlt sie im Eis ab und versetzt vorsichtig mit so viel Äther, bis eine Trübung entstanden ist. Bei ruhigem Stehen im Eis scheidet sich das d-resp. 1-Chlorid in Form von feinen, leuchtend roten Nadeln aus, wobei sich die Lösung klärt. Die Krystalle werden möglichst rasch abgesaugt und sofort auf Ton in den Exsiccator gebracht. Erst nachdem sie gänzlich von den letzten Spuren des Lösungs- resp. Fällungsmittels befreit sind, können sie an der Luft liegen, ohne zu zerfliessen. Das Salz enthält eine Molekel Krystallwasser.

a) d-Chlorid: 14,110 mgr Subst. gaben 11,04 mgr AgCl.

$$
\begin{array}{ll}
5,570 \text { mgr Subst. gaben } 0,7546 \mathrm{~cm}^{3} \text { (korr.) } \mathrm{N}_{2}\left(16^{0}, 723 \mathrm{~mm}\right. \text { ) } \\
\mathrm{C}_{9} \mathrm{H}_{25} \mathrm{O}_{2} \mathrm{~N}_{4} \mathrm{Cl}_{2} \mathrm{Co}+\mathrm{H}_{2} \mathrm{O} \quad \begin{array}{lll}
\text { Ber. } \mathrm{N} 15,22 & \mathrm{Cl} 19,27 \% \\
& \text { Gef. , } 15,23 \quad \text {, } 19,36 \%
\end{array}
\end{array}
$$

b) 1-Chlorid: 55,80 mgr Subst. gaben 28,30 mgr $\mathrm{CoSO}_{4}$

$3,00 \mathrm{mgr}$ Subst. gaben $0,400 \mathrm{~cm}^{3}$ (korr.) $\mathrm{N}_{2}\left(170^{\circ}, 726 \mathrm{~mm}\right)$

$$
\begin{array}{rrr}
\mathrm{C}_{9} \mathrm{H}_{23} \mathrm{O}_{2} \mathrm{~N}_{4} \mathrm{Cl}_{2} \mathrm{Co}+\mathrm{H}_{2} \mathrm{O} & \text { Ber. } \mathrm{N} 15,22 \text { Co } 16,02 \% \\
& \text { Gef. , } 14,99 \text {, } 15,89 \% .
\end{array}
$$

Polarisationen.

d-Chlorid: $[\alpha]_{\mathrm{D}}=+543^{\circ} ;[\mathrm{M}]_{\mathrm{D}}=+2107^{\circ}$.

1-Chlorid: $[a]_{\mathrm{D}}=-550^{\circ} ;[\mathrm{M}]_{\mathrm{D}}=-2134^{\circ}$. 
Bromide: d-resp. I- $\left[\mathrm{C}_{5} \mathrm{H}_{7} \mathrm{O}_{2} \mathrm{Co} \mathrm{C}_{2}\right] \mathrm{Br}_{2}+1 \mathrm{H}_{2} \mathrm{O}$

Sowohl diese Salze wie auch die weiter unten beschriebenen entsprechenden Rhodanide, Perchlorate und Persulfate, werden aus den konzentrierten Lösungen der aktiven Chloride durch doppelten Umsatz erhalten. Es ist dabei nicht notwendig, die Chloride in fester Form zu fassen; man vertährt vielmehr wie folgt. Das fein gepulverte d-resp. 1-Jodid wird mit frischem, noch feuchtem Silberchlorid im Überschuss verrieben und eine kurze Zeit im Dunkeln stehen gelassen. Hierauf versetzt man die Masse mit ungefähr dem gleichen Volumen heissem Wasser und rührt gut um, worauf die tief orangerote Lösung des gebildeten aktiven Chlorides abgesaugt wird; der Niederschlag wird auf dem Filter mit einigen Tropfen heissen Wassers gewaschen.

Die vereinigten Filtrate werden nun mit geeigneten, fein pulverisierten Fällungsmitteln (Kaliumbromid, Kaliumrhodanid etc.) verrieben, wobei ein Niederschlag entsteht, den man nachher umkrystallisiert.

Auf diese Weise erhält man bei Anwendung von Natriumbromid als Fällungsmittel die aktiven Bromide als klein krystallinische, lebhaft rote Niederschläge. Nach dem Umkrystallisieren aus siedendem Alkohol stellen die beiden Bromide dünne, leuchtend rote bis granatrote Prismen (Nadeln) dar, welche in ihrer Löslichkeit in Aceton, Wasser und Alkohol etwa in der Mitte zwischen Jodiden und Chloriden stehen. Auch dieses Salz enthält eine Molekel Krystallwasser.

d-Bromid: 13,00 mgr Subst. gaben 10,70 mgr $\mathrm{AgBr}$

7,60 mgr Subst. gaben $0,823 \mathrm{~cm}^{3} \mathrm{~N}_{2}\left(13^{0}, 727 \mathrm{~mm}\right)$

$\begin{array}{lll}\mathrm{C}_{9} \mathrm{H}_{23} \mathrm{O}_{22} \mathrm{~N}_{4} \mathrm{Br}_{2} \mathrm{Co}+\mathrm{H}_{2} \mathrm{O} & \text { Ber. N } 12,21 & \text { Br } 34,97 \% \\ & \text { Gef. , } 12,37 \quad, 35,03 \%\end{array}$

I-Bromid: 57,80 mgr. Subst. gaben 19,60 mgr CoSO

6,145 mgr Subst. gaben $0,690 \mathrm{~cm}^{3} \mathrm{~N}_{2}\left(16^{0}, 715 \mathrm{~mm}\right)$

$\mathrm{C}_{9} \mathrm{H}_{23} \mathrm{O}_{2} \mathrm{~N}_{4} \mathrm{Br}_{2} \mathrm{Co}+\mathrm{H}_{2} \mathrm{O}$ Ber. $\mathrm{N} 12,21$ Co $12,90 \%$

Polarisationen.

Gef. , $12,50,, 12,90 \%$.

d- resp. 1-Bromid: $[\alpha]_{\mathrm{D}}= \pm 460^{\circ} ;[\mathrm{M}]_{1)}= \pm 2102^{\circ}$.

Rhodanide: d-resp.1- $\left[\mathrm{C}_{5} \mathrm{H}_{7} \mathrm{O}_{2} \mathrm{Co} \mathrm{en}_{2}\right]\left(\mathrm{SCN}_{2}+2 \mathrm{H}_{2} \mathrm{O}\right.$

Die durch Fällen konzentrierter Lösungen der aktiven Chloride mit festem Kaliumrhodanid erhaltenen Niederschläge werden aus Alkohol umkrystallisiert; die beiden aktiven Rhodanide stellen 
lebhaft rote kleine Kryställchen dar und sind sehr leicht löslich in Aceton, Alkohol und Wasser. Sie enthalten zwei Molekel Krystallwasser.

d-Rhodanid: $62,70 \mathrm{mgr}$ Subst. gaben 24,48 $\mathrm{mgr} \mathrm{CoSO}_{4}$ 3,280 mgr Subst. gaben $0,588 \mathrm{~cm}^{3} \mathrm{~N}_{\dot{z}}\left(16^{0}, 726 \mathrm{~mm}\right)$

$$
\begin{array}{lll}
\mathrm{C}_{11} \mathrm{H}_{23} \mathrm{O}_{2} \mathrm{~N}_{6} \mathrm{~S}_{2} \mathrm{Co}+2 \mathrm{H}_{2} \mathrm{O} & \text { Ber. } \mathrm{N} 21,04 & \text { Co } 14,73 \% \\
& \text { Gef. , 20,23 „14,85\% }
\end{array}
$$

1-Rhodanid: 56,22 mgr Subst. gaben 21,58 mgr $\mathrm{CoSO}_{4}$ 5,655 mgr Subst. gaben $1,078 \mathrm{~cm}^{3} \mathrm{~N}_{2}\left(17^{0}, 715 \mathrm{~mm}\right)$

$$
\begin{aligned}
\mathrm{C}_{11} \mathrm{H}_{23} \mathrm{O}_{2} \mathrm{~N}_{6} \mathrm{~S}_{2} \mathrm{Co}+2 \mathrm{H}_{2} \mathrm{O} & \text { Ber. } \mathrm{N} 21,04 \text { Co } 14,73 \% \\
& \text { Gef. "2 } 21,11 \text {, } 14,60 \%
\end{aligned}
$$

Polarisationen.

d- resp. I-Rhodanid: $[a]_{\mathrm{D}}= \pm 533^{\circ} ;[\mathrm{M}]_{\mathrm{D}}= \pm 2107^{\circ}$.

$$
\text { Perchlorate: d-resp. } l\left[\mathrm{C}_{5} \mathrm{H}_{7} \mathrm{O}_{2} \mathrm{Coen}_{2}\right]\left(\mathrm{ClO}_{4}\right)_{2}
$$

Diese Salze werden aus den entsprechenden Chloridlösungen mittelst Natriumperchlorat ausgefällt; aus Alkohol umkrystallisiert, bilden sie schöne, granatrote Prismen, die kein Krystallwasser enthalten und ähnliche Löslichkeitsverhältnisse aufweisen wie etwa die aktiven Bromide.

d-Perchlorat: 52,100 mgr Subst. gaben 17,00 mgr $\mathrm{CoSO}_{4}$

$9,852 \mathrm{mgr}$ Subst. gaben $1,025 \mathrm{~cm}^{3} \mathrm{~N}_{2}\left(16^{0}, 722 \mathrm{~mm}\right)$

$$
\begin{array}{lll}
\mathrm{C}_{9} \mathrm{H}_{23} \mathrm{O}_{10} \mathrm{~N}_{4} \mathrm{Cl}_{2} \mathrm{Co} & \text { Ber. Co } 12,33 & \mathrm{~N} 11,73 \% \\
& \text { Gef. , } 12,40 & \text {, } 11,67 \%
\end{array}
$$

1-Perchlorat: 44,230 mgr Subst. gaben 14,44 mgr $\mathrm{CoSO}_{4}$ 5,785 mgr Subst. gaben $0,594 \mathrm{~cm}^{3} \mathrm{~N}_{2}\left(16^{0}, 723 \mathrm{~mm}\right)$

$$
\begin{aligned}
& \mathrm{C}_{9} \mathrm{H}_{23} \mathrm{O}_{10} \mathrm{~N}_{4} \mathrm{Cl}_{2} \mathrm{Co} \quad \text { Ber. Co 12,33 N 11,73\% } \\
& \text { Gef. , } 12,51, \quad, 11,74 \% \text {. }
\end{aligned}
$$

Polarisationen.

d- resp. l-Perchlorat: $[\alpha]_{I)}= \pm 543^{\circ} ;[M]_{D}= \pm 2107^{\circ}$.

Persulfate: d-resp.l- $\left[\mathrm{C}_{5} \mathrm{H}_{7} \mathrm{O}_{2} \mathrm{Co} \mathrm{en}_{2}\right] \mathrm{S}_{2} \mathrm{O}_{8}$

Zur Darstellung dieser Salze werden die entsprechenden Chloridlösungen mit Ammoniumpersulfat gefällt. Die erhaltenen Niederschläge werden in diesem Falle aus 60-proz. Alkohol umkrystallisiert, weil die Persulfate dieser Reihen in absolutem Alkohol nahezu unlöslich sind, und in heissem Wasser der Racemisation anheimfallen. 
Die schönen, granatroten Krystalle der beiden Persulfate sind wasserfrei.

d-Persulfat: 40,00 mgr Subst. gaben 13,40 $\mathrm{mgr} \mathrm{CoSO}_{4}$

5,325 mgr Subst. gaben $0,586 \mathrm{~cm}^{3} \mathrm{~N}_{2}\left(20^{\circ}, 715 \mathrm{~mm}\right)$

$$
\begin{aligned}
& \mathrm{C}_{9} \mathrm{H}_{23} \mathrm{O}_{10} \mathrm{~N}_{4} \mathrm{~S}_{2} \mathrm{Co} \quad \text { Ber. } \mathrm{N} 11,89 \text { Co } 12,61 \% \\
& \text { Gef. , } 11,70,12,74 \%
\end{aligned}
$$

1-Persulfat: 53,205 mgr Subst. gaben $17,70 \mathrm{mgr}$. $\mathrm{CoSO}_{4}$

$5,60 \mathrm{mgr}$ Subst. gaben $0,578 \mathrm{~cm}^{3} \mathrm{~N}_{2}\left(16^{0}, 723 \mathrm{~mm}\right)$

$$
\begin{aligned}
& \mathrm{C}_{9} \mathrm{H}_{23} \mathrm{O}_{10} \mathrm{~N}_{4} \mathrm{~S}_{2} \mathrm{Co} \text { Ber. N 11,89 Co } 12,61 \% \\
& \text { Gef. , } 11,91 \quad, 12,65 \% \text {. }
\end{aligned}
$$

Polarisationen.

$$
\begin{aligned}
& \text { d-Persulfat: }[\alpha]_{\mathrm{D}}=+433^{\circ} ;[\mathrm{M}]_{\mathrm{D}}=+2040^{\circ} \\
& \text { l-Persulfat: }[\alpha]_{\mathrm{D}}=-480^{\circ} ;[\mathrm{M}]_{\mathrm{D}}=-2261^{\circ} \\
& \text { Nitrate: d-resp. } 1-\left[\mathrm{C}_{5} \mathrm{H}_{7} \mathrm{O}_{2} \mathrm{Co} \mathrm{en}_{2}\right]\left(\mathrm{NO}_{3}\right)_{2}
\end{aligned}
$$

Reine aktive Jodide werden in absolutem Alkohol gelöst und mit berechneten Mengen Silbernitrat in ebenfalls absolutalkoholischer Lösung umgesetzt. Die Lösungen der gebildeten Nitrate werden abgesaugt, im Eis gekühlt und vorsichtig mit absolutem Äther versetzt, bis eine starke Trübung eingetreten ist. Nach einigen Minuten krystallisieren schöne rote Nadeln der aktiven Nitrate aus, wobei sich die Flüssigkeit klärt. Die rasch abgesaugten Krystalle müssen eine Zeit lang im Exsikkator gehalten werden, um von Alkohol- und Ätherspuren gänzlich befreit zu werden, sonst zerfliessen die Salze an der Luft. Die aktiven Nitrate enthalten kein Krystallwasser, und sind spielend löslich in Wasser, Alkohol und Aceton.

d-Nitrat: 5,655 mgr Subst. gaben $1,057 \mathrm{~cm}^{3} \mathrm{~N}_{2}\left(17,5^{\circ}, 715 \mathrm{~mm}\right)$

$$
\begin{array}{ll}
\mathrm{C}_{9} \mathrm{H}_{23} \mathrm{O}_{8} \mathrm{~N}_{6} \mathrm{Co} & \text { Ber. } \mathrm{N} 20,85 \% \\
& \text { Gef. " } 20,65 \%
\end{array}
$$

1-Nitrat: 5,815 mgr Subst. gaben $1,102 \mathrm{~cm}^{3} \mathrm{~N}_{2}\left(16^{0}, 724 \mathrm{~mm}\right)$

$$
\begin{aligned}
& \mathrm{C}_{y} \mathrm{H}_{23} \mathrm{O}_{8} \mathrm{~N}_{6} \mathrm{Co} \quad \text { Ber. } \mathrm{N} 20,85 \% \\
& \text { Gef. „2, } 21,33 \%
\end{aligned}
$$

Polarisationen.

$$
\text { d- resp. I-Nitrat: }[\alpha]_{D}= \pm 550^{\circ} ;[M]_{D}= \pm 2217^{\circ} .
$$

II. Racemische Propionylacetonato-diäthylendiaminkobaltireihe und ihre optisch-aktiven Formen.

$$
\text { W. Karrer. }
$$

\section{Ausgangsmaterial.}

Zur Darstellung der racemischen, der rechts- und der linksdrehenden Propionylacetonato-reihe dienten die racemischen, 
die rechts- und die linksdrehenden Chloro-aquo-diäthylendiaminkobaltisalze, deren Gewinnung bereits geschildert worden ist.

Das Propionylaceton wurde in Anlehnung an die Vorschrift von Claisen und Ekehardt ${ }^{1}$ ) dargestellt; es siedete bei $158^{\circ}$.

Salze der racemischen

Propionylacetonato-diäthylendiamin-kobaltireihe.

$$
\text { J o did: }\left[\mathrm{C}_{6} \mathrm{H}_{9} \mathrm{O}_{2} \mathrm{Co} \mathrm{en}_{2}\right] \mathrm{J}_{2}+1 \mathrm{H}_{2} \mathrm{O}
$$

10 gr reines racemisches Chloro-aquo-diäthylendiamin-kobaltibromid werden in $20 \mathrm{~cm}^{3} 10$-proz. Kalilauge und $15-20 \mathrm{~cm}^{3}$ Wasser gelöst und mit 3 gr Propionylaceton, gelöst in $20 \mathrm{~cm}^{3}$ Alkohol, versetzt. Man erwärmt 1 Minute lang auf $40^{\circ}$, wobei die anfänglich violettrote Lösung tief orangerot wird, filtriert und versetzt das Filtrat mit 10 gr fein gepulvertem Kaliumjodid. Das Jodid der racemischen Propionylacetonatoreihe fällt sofort aus und wird nach dem Umkrystallisieren aus heissem Wasser rein erhalten. Auf Zusatz von weiteren 5 gr Kaliumjodid zur Mutterlauge erhält man noch etwas Jodid. Gesamtausbeute beträgt 9-10 gr. Das reine Salz krystallisiert aus Wasser in prachtvoll ausgebildeten granatroten Prismen, welche eine Molekel Krystallwasser enthalten. $100 \mathrm{~cm}^{3}$ Wasser lösen 2,1 gr Jodid bei $15^{\circ}$ und 21 gr bei $60^{\circ}$.

$$
\begin{aligned}
& 0,1228 \text { gr Subst. gaben } 0,0340 \mathrm{gx}^{\mathrm{CoSO}_{4}} \\
& \text { 43,91 mgr Subst. gaben 36,33 mgr AgJ } \\
& \text { 9,36 mgr Subst. gaben } 0,827 \mathrm{~cm}^{3} \mathrm{~N}_{2}\left(9,5^{0}, 725 \mathrm{~mm}\right) \\
& 0,1546 \text { gr Subst. gaben } 0,0048 \mathrm{gr}_{2} \mathrm{O} \\
& \mathrm{C}_{10} \mathrm{H}_{25} \mathrm{O}_{2} \mathrm{~N}_{4} \mathrm{~J}_{2} \mathrm{Co}+\mathrm{H}_{2} \mathrm{O} \\
& \text { Ber. N } \quad 9,93 \quad J 45,00 \quad \text { Co } 10,45 \quad \mathrm{H}_{2} \mathrm{O} \quad 3,19 \% \\
& \text { Gef. , } 10,22 \quad, 44,72 \quad, 10,53 \quad, 3,10 \% \text {. } \\
& \text { Bromid: }\left[\mathrm{C}_{6} \mathrm{H}_{9} \mathrm{O}_{2} \mathrm{Co} \mathrm{en}_{2}\right] \mathrm{Br}_{2}+1 \mathrm{H}_{2} \mathrm{O}
\end{aligned}
$$

$5 \mathrm{gr}$ Jodid werden in wenig warmem Wasser gelöst, mit einem kleinen Überschuss an frisch gefälltem Silberbromid versetzt und etwa eine halbe Stunde lang geschüttelt. Man saugt hierauf die Lösung von überschüssigem Silberbromid und gebildetem Silberjodid ab und engt das Filtrat im Wasserbade stark ein. Nach längerem Stehen der abgekühlten Lösung scheidet sich das

1) B. 22, 1009 (1889). 
Bromid in granatroten Krystallen aus; es enthält eine Molekel Krystallwasser.

0,0515 gr Subst. gaben 0,0171 gr $\operatorname{CoSO}_{4}$

$40,71 \mathrm{mgr}$ Subst. gaben $32,54 \mathrm{mgr} \mathrm{AgBr}$

0,1958 gr Subst. gaben 0,0074 gr $\mathrm{H}_{2} \mathrm{O}\left(140-150^{0}\right.$;

$\mathrm{C}_{10} \mathrm{H}_{25} \mathrm{O}_{2} \mathrm{~N}_{4} \mathrm{Br}_{2} \mathrm{Co}+\mathrm{H}_{2} \mathrm{O}$

Ber. Br 34,00 Co $12,54 \quad \mathrm{H}_{2} \mathrm{O} 3,83 \%$

Gef. ", 34,02 , $12,63 \quad$, $3.78 \%$.

Chlorid: $\left[\mathrm{C}_{6} \mathrm{H}_{9} \mathrm{O}_{2} \mathrm{Coen}_{9}\right] \mathrm{Cl}_{2}+\mathrm{I} \mathrm{H}_{2} \mathrm{O}$

5 gr Jodid werden in wenig warmem Wasser gelöst und eine halbe Stunde lang mit einem kleinen Überschuss an frisch bereitetem Silberchlorid geschüttelt. Die abfiltrierte Lösung wird bis zur Syrupdicke eingeengt und dann ruhig stehen gelassen. Nach mehreren Stunden scheidet sich das Chlorid aus, doch sind die Krystalle nicht schön ausgebildet. Das granatrote Salz ist. sehr leicht löslich in Wasser und krystallisiert, gleich dem Jodid und dem Bromid, mit einer Molekel Wasser.

0,0694 gr Subst. gaben 0,0283 gr $\mathrm{CoSO}_{4}$

25,10 mgr Subst. gaben 18,88 mgr $\mathrm{AgCl}$

0,1550 gr Subst. gaben 0,0071 gr $\mathrm{H}_{2} \mathrm{O}$

$\mathrm{C}_{10} \mathrm{H}_{25} \mathrm{O}_{2} \mathrm{~N}_{4} \mathrm{Cl}_{2} \mathrm{Co}+\mathrm{H}_{2} \mathrm{O}$

Ber. Cl 18,60 Co $15,47 \quad \mathrm{H}_{2} \mathrm{O} 4,73 \%$

Gef. ", 18,61 , $15,51 \quad, \quad 4.58 \%$

Nitrat: $\left[\mathrm{C}_{6} \mathrm{H}_{9} \mathrm{O}_{2} \mathrm{Coen}_{2}\right]\left(\mathrm{NO}_{3}\right)_{2}+1 \mathrm{H}_{2} \mathrm{O}$

Man setzt 5 gr Jodid, gelöst in wenig warmem Wasser, mit einer wässerigen Lösung von $3,1 \mathrm{gr}$ Silbernitrat um. Die Lösung wird vom abgeschiedenen Silberjodid abfiltriert, das Filtrat mit einigen Körnchen Kochsalz versetzt, um das etwa noch als Nitrat vorhandene Silber zu fällen, von neuem filtriert und eingeengt. Bei längerem Stehen der Lösung scheidet sich das Nitrat in Form von grossen granatroten Krystallen aus.

$$
\begin{aligned}
& 0,0857 \text { gr Subst. gaben } 0,0310 \mathrm{gr} \mathrm{CoSO}_{4} \\
& \text { 5,41 mgr Subst. gaben } 0,921 \mathrm{~cm}^{3} \mathrm{~N}_{2}\left(9,5^{0}, 721 \mathrm{~mm}\right. \text {; } \\
& 0,1610 \text { gr Subst. gaben } 0,0069 \text { gr } \mathrm{H}_{2} \mathrm{O} \\
& \mathrm{C}_{20} \mathrm{H}_{25} \mathrm{O}_{8} \mathrm{~N}_{6} \mathrm{Co}+\mathrm{H}_{2} \mathrm{O} \\
& \text { Ber. N 19,36 Co } 13,58 \quad \mathrm{H}_{2} \mathrm{O} 4,15 \% \\
& \text { Gef. , } 19,52 \quad, 13.76 \quad, \quad 4,28 \%
\end{aligned}
$$




\section{$-125-$ \\ Sulfat: $\left[\mathrm{C}_{6} \mathrm{H}_{9} \mathrm{O}_{2} \mathrm{Coen}_{2}\right] \mathrm{SO}_{4}$}

Man schüttelt die konzentrierte, wässerige Lösung von 5 gr Jodid mit einem kleinen Überschuss an frisch bereitetem Silbersulfat etwa eine halbe Stunde lang. Die hierauf abfiltrierte, wenn nötig mit einigen Körnchen Kochsalz entsilberte und wiederum filtrierte Lösung wird eingeengt und ruhig stehen gelassen. Das Sulfat krystallisiert in langen, roten Nadeln aus, welche wasserfrei sind.

0,0588 gr Subst. gaben $0,0237 \mathrm{gr} \mathrm{CoSO}_{4}$

4,86 mgr Subst. gaben $0,6115 \mathrm{~cm}^{3} \mathrm{~N}_{2}\left(12,8^{\circ}, 725 \mathrm{~mm}\right)$

$$
\begin{array}{lll}
\mathrm{C}_{10} \mathrm{H}_{25} \mathrm{O}_{6} \mathrm{~N}_{4} \mathrm{SCo} & \text { Ber. N } 14,43 & \text { Co } 15,19 \% \\
& \text { Gef. , } 14,39 & \text { " } 15,33 \%
\end{array}
$$

Rhodanid: $\left[\mathrm{C}_{6} \mathrm{H}_{9} \mathrm{O}_{2}\right.$ Co en 2$](\mathrm{SCN})_{2}$

Sowohl dieses Salz, wie auch die weiter unten beschriebenen Persulfate und Perchlorate werden auf dem Umweg über das Chlorid gewonnen, das jedoch nicht isoliert $\mathrm{zu}$ werden braucht.

Man bereitet, wie oben angegeben, eine Chloridlösung aus 8 gr Jodid durch Umsatz mit Silberchlorid, engt sie ein und versetzt sie mit festem Kaliumrhodanid. Der gebildete rote Niederschlag wird abgesaugt und aus heissem Wasser umkrystallisiert. Die kleinen granatroten Rhodanidkrystalle sind wasserfrei.

0,1334 gr Subst. gaben 0,0507 gr $\mathrm{CoSO}_{4}$

4,48 mgr Subst. gaben $0,804 \mathrm{~cm}^{3} \mathrm{~N}_{2}\left(9,5^{0}, 725 \mathrm{~mm}\right)$

$$
\begin{array}{llr}
\mathrm{C}_{12} \mathrm{H}_{25} \mathrm{O}_{2} \mathrm{~N}_{6} \mathrm{~S}_{2} \mathrm{Co} & \text { Ber. } \mathrm{N} \mathrm{20,58} \text { Co } 14,44 \% \\
& \text { Gef. , } 20,68 \quad \text { " } 14,46 \%
\end{array}
$$

Perchlorat: $\left[\mathrm{C}_{6} \mathrm{H}_{9} \mathrm{O}_{2} \mathrm{Coen}_{2}\right]\left(\mathrm{ClO}_{4}\right)_{2}$

Die aus 8 gr Jodid gewonnene konzentrierte Chloridlösung wird mit Natriumperchlorat verrieben, wobei sich ein reichlicher roter Niederschlag des Perchlorates bildet. Nach zweimaligem Umkrystallisieren aus Wasser erhält man das reine Salz in schön ausgebildeten granatroten Krystallen, welche keinWasser enthalten.

0,0976 gr Subst. gaben 0,0308 gr $\mathrm{CoSO}_{4}$ $9,325 \mathrm{mgr}$ Subst. gaben $0,970 \mathrm{~cm}^{3} \mathrm{~N}_{2}\left(14,5^{0}, 722,3 \mathrm{~mm}\right)$

$$
\begin{aligned}
& \mathrm{C}_{10} \mathrm{H}_{25} \mathrm{O}_{10} \mathrm{~N}_{4} \mathrm{Cl}_{2} \mathrm{Co} \text { Ber. N 11,41 Co } 12,01 \% \\
& \text { Gef. , } 11,48 \quad, 12,00 \% \\
& \text { Persulfat: }\left[\mathrm{C}_{6} \mathrm{H}_{9} \mathrm{O}_{2} \mathrm{Co}_{2}\right] \mathrm{S}_{2} \mathrm{O}_{8}
\end{aligned}
$$

Dieses Salz fällt auf Zusatz von Ammoniumpersulfat zur Chloridlösung aus. 
Man löst den abfiltrierten Niederschlag in heissem Wasser auf und lässt erkalten: es scheiden sich kleine orangerote Nadeln des reinen Persulfates aus. Es ist das am wenigsten lösliche Salz der Reihe; es enthält kein Krystallwasser.

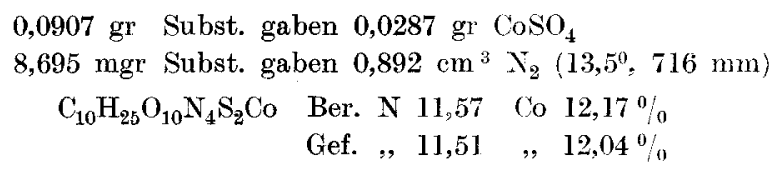

Salze der optisch-aktiven

Propionylacetonato-diäthylendiamin-kobaltireihen.

Jodide: d-resp.l- $\left[\mathrm{C}_{6} \mathrm{H}_{9} \mathrm{O}_{2} \mathrm{Co} \mathrm{en}_{2}\right] \mathrm{J}_{2}+1 \mathrm{H}_{2} \mathrm{O}$

Man löst 10 gr d- resp. l-Chloro-aquo-diäthylendiaminkobaltibromid in $20 \mathrm{~cm}^{3}$ 10-proz Kalilauge und $20 \mathrm{~cm}^{3}$ Wasser, fügt $3 \mathrm{gr}$ Propionylaceton, gelöst in $20 \mathrm{~cm}^{3}$ Alkohol, hinzu und erwärmt eine Minute lang auf $40^{\circ}$. Die nunmehr tief orangerot gefärbte Lösung wird filtriert und mit $5 \mathrm{gr}$ fein gepulvertem Kaliumjodid versetzt, worauf man sofort vom gebildeten Niederschlag - zur Hauptsache Hydroxo-aquo-diäthylendiamin-kobaltisalz - abfiltriert. Das Filtrat wird mit weiteren $5 \mathrm{gr}$ Kaliumjodid versetzt, wobei von neuem ein Niederschlag entsteht, welchen man nach fünf Minuten absaugt; er besteht hauptsächlich aus racemischem Jodid der Propionylacetonato-reihe. Man lässt nun das Filtrat bei Zimmertemperatur stehen und isoliert Krystallisationen nach ein, zwei, vier und sechs Tagen, den Rest nach drei Wochen.

Man vereinigt ungefähr gleichdrehende Fraktionen von vier bis fünf Ansätzen (also entsprechend 40--50 gr Ausgangsmaterial) und unterwirft sie weiterer fraktionierter Krystallisation aus Wasser, u. s. f. Man erhält schliesslich (nach etwa drei Fraktionsfolgen) schöne, grosse, granatrote Säulen der reinen aktiven Jodide; allerdings wurde das Drehungsvermögen der linksdrehenden Form etwas niedriger gefunden, als jenes der d-Form.

Polarisationen : 0,1 -proz. wässerige Lösungen, Schichtlänge $10 \mathrm{~cm}$.

d-Jodid: $\alpha_{\mathrm{D}}=+0,465 ; \quad[\alpha]_{\mathrm{D}}=+465^{\circ} ; \quad[\mathrm{M}]_{\mathrm{D}}=+2623^{\circ} ;$

1. Jodid: $\alpha_{\mathrm{D}}=-0,434 ;[\alpha]_{\mathrm{D}}=+434^{\circ} ; \quad[\mathrm{M}]_{\mathrm{D}}=-2448^{\circ}$.

Diese Drehungswerte sanken bei ca. vier Monate langem Lagern der trockenen Salze auf $[\alpha]_{D}=+402^{0}$ resp. $-390^{\circ}$. 
Die leicht löslichen aktiven Jodide enthalten eine Molekel Krystallwasser.

a) d-Jodid: 0,0888 gr Subst. gaben 0,0250 gr $\mathrm{CoSO}_{4}$

0,1601 gr Subst. gaben $0,0050 \mathrm{gr} \mathrm{H}_{2} \mathrm{O}$

39,32 mgr Subst. gaben $32,80 \mathrm{mgr}$ AgJ

$$
\begin{aligned}
& \mathrm{C}_{10} \mathrm{H}_{25} \mathrm{O}_{2} \mathrm{~N}_{4} \mathrm{~J}_{2} \mathrm{Co}+\mathrm{H}_{2} \mathrm{O} \text {. Ber. J 45,00 Co } 10,45 \quad \mathrm{H}_{2} \mathrm{O} \quad 3,19 \% \\
& \text { Gef. } \quad 45,09 \quad, 10,71 \quad, 3,12 \%
\end{aligned}
$$

b) 1-Jodid: 0,0909 gr Subst. gaben 0,0256 gr $\mathrm{CoSO}_{4}$

0,1479 gr Subst. gaben $0,0047 \mathrm{gr}_{2} \mathrm{O}$

42,00 mgr Subst. gaben 35,02 mgr AgJ

$$
\begin{aligned}
& \mathrm{C}_{10} \mathrm{H}_{25} \mathrm{O}_{2} \mathrm{~N}_{4} \mathrm{~J}_{2} \mathrm{Co}+\mathrm{H}_{2} \mathrm{O} \text {. Ber. J } 45,00 \text { Co } 10,45 \quad \mathrm{H}_{2} \mathrm{O} 3,19 \% \\
& \text { Gef. , } 45,07 \quad, 10,71 \quad, 3,19 \%
\end{aligned}
$$

Nitrate: d-resp.1- $\left[\mathrm{C}_{6} \mathrm{H}_{9} \mathrm{O}_{2} \mathrm{Co} \mathrm{en}_{2}\right]\left(\mathrm{NO}_{3}\right)_{2}$

$4 \mathrm{gr}$ d- resp. 1-Jodid, die eine spezifische Drehung von ca. $\pm 400^{\circ}$ hatten, wurden in warmem Wasser gelöst und mit einer wässerigen Lösung von 2,5 gr Silbernitrat umgesetzt. Man filtriert das abgeschiedene Silberjodid ab, entsilbert das Filtrat wenn nötig mit einigen Körnchen Kochsalz, filtriert nochmals und engt ein. Aus der stark konzentrierten Lösung scheidet sich beim Stehen d- resp. l-Nitrat in Form von grossen, schön ausgebildeten Krystallen aus, welche wasserfrei sind.

Polarisationen: 0,1-proz. Lösungen, Schichtlänge $10 \mathrm{~cm}$

d-Nitrat: $\alpha_{\mathrm{D}}=+0,596 ; \quad[\alpha]_{\mathrm{D}}=+596^{\circ} ; \quad[\mathrm{M}]_{\mathrm{D}}=+2481^{0}$

1-Nitrat: $a_{\mathrm{D}}=-0,591 ; \quad[\alpha]_{\mathrm{D}}=-591^{\circ} ; \quad[\mathrm{M}]_{\mathrm{D}}=-2460^{\circ}$

Drehungswerte nach ca. vier Monaten:

$$
[\alpha]_{\mathrm{D}}=+496^{\circ}, \text { resp. }-511^{\circ}
$$

a) d-Nitrat: 0,1048 gr Subst. gaben 0,0393 gr $\mathrm{CoSO}_{4}$

5,21 mgr Subst. gaben $0,920 \mathrm{~cm}^{3} \mathrm{~N}_{2}\left(12,5^{0}, 732,5 \mathrm{~mm}\right)$

$$
\begin{aligned}
& \mathrm{C}_{10} \mathrm{H}_{25} \mathrm{O}_{8} \mathrm{~N}_{6} \text { Co. Ber. } \mathrm{N} \mathrm{20,19} \text { Co } 14,17 \% \\
& \text { Gef. , 20,35 ", 14,26\% }
\end{aligned}
$$

b) 1-Nitrat: 0,1047 gr Subst. gaben 0,0389 $\mathrm{gr} \mathrm{CoSO}_{4}$

4,765 mgr Subst. gaben $0,841 \mathrm{~cm}^{3} \mathrm{~N}_{2}\left(13,4^{0}, 727,8 \mathrm{~mm}\right)$

$$
\begin{array}{lll}
\mathrm{C}_{10} \mathrm{H}_{25} \mathrm{O}_{8} \mathrm{~N}_{6} \text { Co. } & \text { Ber. } \mathrm{N} 20,19 & \text { Co } 14,17 \% \\
& \text { Gef. "2 20,14 " } 14,13 \%
\end{array}
$$

Perchlorate: d-resp. 1- $\left[\mathrm{C}_{6} \mathrm{H}_{9} \mathrm{O}_{2} \mathrm{Co} \mathrm{en}_{2}\right]\left(\mathrm{ClO}_{4}\right)_{2}$

Zur Darstellung dieser Salze und der weiter unten beschriebenen Persulfate bereitet man zweckmässig zuerst eine Lösung 
der aktiven Chloride, welche dann mit entsprechenden Fällungsmitteln versetzt wird. Die Chloride selber konnten nicht isoliert werden; diese und die Bromide sind so zerfliesslich, dass nicht einmal die Fällung ihrer absolut-alkoholischen Lösungen mit trockenem Äther feste Substanz zu liefern vermag. 5 gr d- resp. l-Jodid werden in wenig warmem Wasser gelöst, mit einem kleinen Überschuss an frisch bereitetem Silberchlorid versetzt und eine halbe Stunde lang geschüttelt. Man saugt von den Silbersalzen ab, engt das Filtrat im Wasserbade ein und fällt es nach dem Erkalten mit Natriumperchlorat. Nach dem Umkrystallisieren der Niederschläge aus heissem Wasser erhält man schön ausgebildete, granatrote Krystalle des d- resp. 1-Perchlorates. Das Salz enthält kein Krystallwasser.

Polarisationen: 0,1-proz. Lösung, Schichtlänge $10 \mathrm{~cm}$.

d-Perchlorat: $\alpha_{\mathrm{D}}=+0,504 ; \quad[\alpha]_{\mathrm{D}}=+504^{\circ} ; \quad[\mathrm{M}]_{\mathrm{D}}=+2476^{\circ}$.

1-Perchlorat: $a_{\mathrm{D}}=-0,496 ;[a]_{\mathrm{D}}=-496^{\circ} ;[\mathrm{M}]_{\mathrm{J}}=-2436^{\circ}$.

Nach vier Monaten betrug das spezifische Drehungsvermögen noch

$$
[\alpha]_{\mathrm{D}}:+434^{0} \text { resp. }-458^{\circ} \text {. }
$$

a) d-Perchlorat: 0,0960 gr Subst. gaben $0,0303 \mathrm{gr} \mathrm{CoSO}_{4}$

7,825 mgr Subst. gaben $0,817 \mathrm{~cm}^{3} \mathrm{~N}_{2}\left(15,8^{\circ}, 716 \mathrm{~mm}\right)$

$$
\begin{array}{lll}
\mathrm{C}_{10} \mathrm{H}_{25} \mathrm{O}_{10} \mathrm{~N}_{4} \mathrm{Cl}_{2} \mathrm{Co} . & \text { Ber. N 11,41 Co } 12,01 \% \\
& \text { Gef. , } 11,63 \quad \text {, } 12,01 \%
\end{array}
$$

b) 1-Perchlorat: $0,0768 \mathrm{gr}_{\mathrm{r}}$ Subst. gaben $0,0243 \mathrm{gr} \mathrm{CoSO}$

$9,660 \mathrm{mgr}$ Subst. gaben $1,005 \mathrm{~cm}^{3} \mathrm{~N}_{2}\left(15,8^{\circ}, 721 \mathrm{~mm}\right)$

$$
\begin{array}{lll}
\mathrm{C}_{10} \mathrm{H}_{25} \mathrm{O}_{10} \mathrm{~N}_{4} \mathrm{Cl}_{2} \mathrm{Co} . & \text { Ber. } \mathrm{N} 11,41 & \text { Co } 12,01 \% \\
& \text { Gef. , } 11,65 \quad, 12,03 \%
\end{array}
$$

$$
\text { Persulfate: d-resp. l- }\left[\mathrm{C}_{6} \mathrm{H}_{9} \mathrm{O}_{2} \mathrm{Coen}_{2}\right] \mathrm{S}_{2} \mathrm{O}_{6}
$$

Man fällt konzentrierte Lösungen von d-resp. l-Chlorid, welche, wie oben, aus d- resp. l-Jodid durch Umsatz mit Silberchlorid gewonnen werden, mit Ammoniumpersulfat und krystallisiert die erhaltenen Niederschläge aus heissem Wasser um. Beim Abkühlen erhält man schwerlösliche orangerote Nadeln der aktiven Persulfate, welche kein Krystallwasser enthalten.

Polarisationen : ${ }^{1},{ }_{30}$-proz. Lösung, Schichtlänge $10 \mathrm{~cm}$.

d-Persulfat: $\alpha_{\mathrm{D}}=+0,159 ; \quad[\alpha]_{\mathrm{D}}=+477^{\circ} ;[\mathrm{M}]_{\mathrm{D}}=+2311^{\circ}$

1.Persulfat: $\alpha_{\mathrm{D}}=-0,164 ;[\alpha]_{\mathrm{D}}=-492^{\circ} ; \quad[\mathrm{M}]_{\mathrm{D}}=-2383^{\circ}$.

Nach ca. vier Monaten sanken diese Werte auf

$$
[\alpha]_{\mathrm{D}}=+404^{\circ} \text { resp. }-420^{\circ} \text {. }
$$


a) d-Persulfat: 0,1185 gr Subst. gaben 0,0379 gr $\mathrm{CoSO}_{4}$

$8,310 \mathrm{mgr}$ Subst. gaben $0,860 \mathrm{~cm}^{3} \mathrm{~N}_{2}\left(13^{0}, 716,5 \mathrm{~mm}\right)$

$$
\begin{array}{lll}
\mathrm{C}_{10} \mathrm{H}_{25} \mathrm{O}_{10} \mathrm{~N}_{4} \mathrm{~S}_{2} \text { Co. } & \text { Ber. N } 11,57 & \text { Co } 12,17 \% \\
& \text { Gef. " } 11,65 \quad \text {, } 12,16 \%
\end{array}
$$

b) 1-Persulfat: $0,0488 \mathrm{gr}$ Subst. gaben 0,0156 $\mathrm{gr} \mathrm{CoSO}_{4}$

6,58 mgr Subst. gaben $0,676 \mathrm{~cm}^{3} \mathrm{~N}_{2}\left(12,4^{0}, 716 \mathrm{~mm}\right)$

$$
\begin{array}{lll}
\mathrm{C}_{10} \mathrm{H}_{25} \mathrm{O}_{10} \mathrm{~N}_{4} \mathrm{~S}_{2} \mathrm{Co} \text {. } & \text { Ber. } \mathrm{N} 11,57 & \text { Co } 12,17 \% \\
& \text { Gef. ", } 11,60 & \text {, } 12,16 \%
\end{array}
$$

\section{Tabelle II.}

Anormale Rotationsdispersion bei d- rsp. l-Propionylacetonato-diäthylen-diaminkobaltijodiden.

\begin{tabular}{|c|c|c|}
\hline \multirow{2}{*}{$\begin{array}{c}\text { Wellenlängen } \\
\mu \mu\end{array}$} & \multicolumn{2}{|c|}{ Spezif. Drehungsvermögen $[\alpha]$} \\
\cline { 2 - 3 } & d-Jodid & 1-Jodid \\
\hline \hline 644 & $+115^{\circ}$ & $-135^{\circ}$ \\
626 & $+186^{\circ}$ & $-190^{\circ}$ \\
607,5 & $+276^{\circ}$ & $-268^{\circ}$ \\
589,3 & $+402^{\circ}$ & $-390^{\circ}$ \\
575 & $+518^{\circ}$ & $-535^{\circ}$ \\
560 & $+630^{\circ}$ & $-645^{\circ}$ \\
554 & $+650^{\circ}$ & $-672^{\circ}$ \\
547,5 & $+600^{\circ}$ & $-628^{\circ}$ \\
542,5 & $+554^{\circ}$ & $-584^{\circ}$ \\
537 & $+440^{\circ}$ & $-480^{\circ}$ \\
532 & $+360^{\circ}$ & $-388^{\circ}$ \\
\hline
\end{tabular}

Zürich, Chemisches Laboratorium der Universität.

9. Dezember 1920. 